\title{
miR-192 suppresses the tumorigenicity of prostate cancer cells by targeting and inhibiting nin one binding protein
}

\author{
JIANTAO SUN ${ }^{1}$, ZHIQIANG FAN ${ }^{2}$, SHUAIQI LU ${ }^{1}$, JINHUI YANG ${ }^{1}$, \\ TONGTONG HAO ${ }^{1}$ and QINGXIANG HUO ${ }^{1}$ \\ ${ }^{1}$ Department of Urology, Luoyang Central Hospital Affiliated to Zhengzhou University, Luoyang, Henan 471002; \\ ${ }^{2}$ Department of Urology, Henan Provincial People's Hospital, Zhengzhou, Henan 450003, P.R. China
}

Received June 25, 2015; Accepted December 10, 2015

DOI: $10.3892 /$ ijmm.2016.2449

\begin{abstract}
Nin one binding (NOB1) protein has been identified as an oncogene in various human cancers, including prostate cancer. MicroRNAs (miRs) have also been recognized as novel regulatory molecules of gene expression. The present study aimed to discover potential miRs that target NOB1 and regulate NOB1 expression in prostate cancer. miR-192, which is an important regulator of numerous cancers, was found to be significantly downregulated in prostate cancer cells. Moreover, we noted that miR-192 overexpression markedly inhibited the proliferation, colony-forming ability, and migratory capacity of the prostate cancer cells. miR-192 overexpression also induced cell cycle arrest in the G1 phase, as shown by flow cytometry. Bioinformatics analysis results revealed that NOB1 was a possible candidate target gene of miR-192. This discovery was further verified through dual-luciferase reporter assay, RT-qPCR, and western blot analysis. We suggest that miR-192 directly regulates the mRNA and protein expression of NOB1. Furthermore, miR-192 inhibited the expression of p38 mitogenactivated protein kinase. The results of our study indicated that miR-192 negatively regulated NOB1 expression and impaired the tumorigenicity of prostate cancer cells. Therefore, we suggest that targeting miR-192 and NOB1 is a novel strategy which will assist in the development of new therapeutics that will be used in the future to prevent and treat prostate cancer.
\end{abstract}

Correspondence to: Dr Jiantao Sun, Department of Urology, Luoyang Central Hospital Affiliated to Zhengzhou University, 288 Zhongzhou Road, Xigong, Luoyang, Henan 471002, P.R. China E-mail: sunjiantaohx@163.com

Abbreviations: NOB1, nin one binding; miRs, microRNAs; miR-192, microRNA-192; MAPK, mitogen-activated protein kinase; 3'-UTR, 3'-untranslated region; MTT, 3-(4,5-dimethylthiazol-2-yl)2,5-diphenyltetrazolium bromide

Key words: prostate cancer, microRNA-192, nin one binding, cell proliferation

\section{Introduction}

Prostate cancer is one of the most common type of malignant tumor which affects males and is the second leading cause of cancer-related mortality among the male gender (1). However, currently available treatments are unable to completely cure advanced prostate cancer, as prostate cancer cells exhibit a high rate of proliferation, as well as high invasion capacity and metastatic ability (2). The underlying mechanism of the tumorigenesis of prostate cancer is not yet fully understood, and as such the development of efficient therapies is impeded. Therefore, the molecular mechanism of prostate cancer must be studied in order to develop novel approaches and agents to prevent and treat prostate cancer.

The nin one binding (NOB1) protein functions as an oncogene which plays an important role in various human cancers $(3,4)$. The human NOB1 gene is located on chromosome $16 \mathrm{q} 22.1$ encodes a $50-\mathrm{kDa}$ protein and is widely expressed in numerous organs, including the lungs, spleen, and liver (5). NOB1 protein is mainly distributed in the nucleus, and this protein is involved in the biogenesis and function of the $20 \mathrm{~S}$ proteasome (6). The important role which NOB1 plays in numerous human cancers, including ovarian cancer (4), hepatocellular carcinoma (7), breast cancer (8), gliomas (9), and lung cancer (10), has been previously investigated. Likewise, the involvement of NOB1 in prostate cancer has also been previously explored: for example, prostate cancer tissues have been shown to express NOB1 in the nucleus, and this expression has been correlated with lymph node metastasis (11). NOB1 expression has been suggested to function as a potential prognostic marker of prostate cancer $(12,13)$. Gene silencing of NOB1 has previously been shown to suppress the malignant transformation of prostate cancer cells (14). Therefore, we posit that targeting NOB1 constitutes a promising approach to preventing and treating prostate cancer.

It has previously been noted that a group of short, non-coding RNAs termed microRNAs (miRs) act as novel regulatory molecules of gene expression (15). miRs are known to negatively regulate the expression of target genes at a post-transcriptional level by binding the 3'-untranslated region (3'-UTR) of mRNA $(15,16)$. Thus, miRs play an important role in the diagnosis, prognosis and treatment of various diseases $(17,18)$. miRs have also been implicated in the pathogenesis of prostate 
cancer; these molecules serve as a novel target in the treatment of prostate cancer (19).

The present study aimed to discover potential miRs that target NOB1 and regulate NOB1 expression in prostate cancer. miR-192 functions as a tumor suppressor in the development and progression of certain human cancers, for example colon (20) and bladder (21) cancers. However, the role of miR-192 in prostate cancer had not previously been examined, to the best of our knowledge. In the present study, we revealed that miR-192 expression was significantly decreased in prostate cancer cells. miR-192 overexpression markedly impaired the tumorigenicity of prostate cancer cells. NOB1 was predicted as a candidate target gene of miR-192 through bioinformatics analysis; this result was confirmed through a dual-luciferase reporter assay. We further demonstrated that miR-192 directly regulated NOB1 expression, and noted that miR-192 overexpression also inhibited the expression of $\mathrm{p} 38$ mitogen-activated protein kinase (MAPK) by suppressing NOB1. Thus, we suggest that miR-192 negatively regulated NOB1 expression in prostate cancer. Targeting miR-192 and NOB1 may thus constitute a novel strategy with which to develop new therapeutics for prostate cancer.

\section{Materials and methods}

Cell cultures. The human prostate cancer lines PC-3 and DU145 were both purchased from the The Cell Bank of Type Culture Collection of the Chinese Academy of Sciences (Shanghai, China). Human prostate epithelial RWPE-1 cells were purchased from the American Type Culture Collection (ATCC; Manassas, VA, USA). PC-3 and DU145 cells were grown in RPMI-1640 medium with 10\% fetal bovine serum (FBS) and $1 \%$ streptomycin-penicillin (all from Invitrogen, Carlsbad, CA, USA). The RWPE-1 cells were grown in keratinocyte serum-free media containing $0.5 \%$ streptomycin-penicillin (both from Invitrogen). All cells were cultured in a $\mathrm{CO}_{2}$ incubator (Heracell 2401; Thermo Fisher Scientific, Waltham, MA, USA) containing $5 \% \mathrm{CO}_{2}$ at $37^{\circ} \mathrm{C}$.

Cell transfection. The cells were grown in a 6-well tissue-culture plate at a density of $1 \times 10^{6}$ cells/well. After the cells reached $60-80 \%$ confluence, the miR-192 mimics and the non-specific miR control (miR NC) synthesized by the Shanghai GenePharma Co., Ltd. (Shanghai, China) were transfected into the cells using Lipofectamine ${ }^{\circledR} 2000$ (Invitrogen) at a final concentration of $50 \mathrm{nM}$, in accordance with the manufacturer's recommendations. After $48 \mathrm{~h}$ of transfection, gene expression was detected through reverse-transcription quantitative polymerase chain reaction (RT-qPCR) and western blot analysis.

$R T$-qPCR analysis. Total RNA was obtained from the cells using an miRNeasy mini kit (Qiagen, Dusseldorf, Germany) in accordance with the manufacturer's instructions. In RT-qPCR mRNA analysis, total RNA was reverse transcribed using M-MLV reverse transcriptase (Clontech Laboratories, Palo Alto, CA, USA). Complementary DNA was synthesized using an miScript reverse transcription kit (Qiagen). qPCR experiments were conducted using an ABI 7500 Real-Time PCR system (Applied Biosystems Life Technologies, Carlsbad, CA, USA) with a SYBR Premix Ex $\mathrm{Taq}^{\mathrm{TM}}$ II commercial kit
(Takara Bio, Dalian, China). Glyceraldehyde 3-phosphate dehydrogenase (GAPDH) and U6 snRNA were used as the internal control for relative gene expression quantification using the $2^{-\Delta \Delta \mathrm{Ct}}$ method.

Western blot analysis. Proteins were extracted from each sample of the cells and quantified using a bicinchoninic acid kit (Beyotime Institute of Biotechnology, Haimen, China). Equivalent amounts of protein $(25 \mu \mathrm{g})$ were loaded onto $12.5 \%$ sodium dodecyl sulfate-polyacrylamide gel electrophoresis and transferred to a nitrocellulose membranes (Miltenyi Biotec, Auburn, CA, USA). After the membranes were blocked with $2.5 \%$ skim milk in Tris-buffered saline (TBS) at $37^{\circ} \mathrm{C}$ for $1 \mathrm{~h}$, the membranes were blotted with primary antibodies, namely anti-NOB1 (sc-160594), anti-p38 MAPK (sc-6176), and anti-GAPDH (sc-48166) antibodies (Santa Cruz Biotechnology, Inc., Santa Cruz, CA, USA) at $4^{\circ} \mathrm{C}$ overnight. After the membranes were washed with TBS and Tween-20, horseradish peroxidase-conjugated secondary antibodies (1:2,000; sc-2768; Santa Cruz Biotechnology, Inc.) were applied for $1 \mathrm{~h}$ at room temperature. The membranes were incubated for $1 \mathrm{~h}$ and subjected to an enhanced chemiluminescence detection system (Amersham Biosciences, Little Chalfont, UK). The blots were developed using an enhanced chemiluminescence detection kit (Amersham Biosciences). The gray value of each protein band was quantified using Image-Pro Plus 6.0 software (Media Cybernetics, Inc., Rockville, MD, USA).

Cell proliferation assay. Human prostate cancer cells were seeded into a 96-well plate at a density of $1 \times 10^{3}$ cells/well and cultured for $24 \mathrm{~h}$. The cells were transfected with $50 \mathrm{nM}$ of miR-192 mimics or miR NC for $48 \mathrm{~h}$. Cell proliferation was detected using a 3-(4,5-dimethylthiazol-2-yl)-2,5-diphenyltetrazolium bromide (MTT) assay. In brief, $5 \mathrm{mg} / \mathrm{ml}$ MTT solution (SigmaAldrich, St. Louis, MO, USA) was added at $20 \mu \mathrm{l} /$ well to the cell cultures. After $4 \mathrm{~h}$ of incubation, the medium was discarded, and the formazan product was dissolved with dimethyl sulfoxide (200 $\mu \mathrm{l} /$ well). The optical density of each well was detected at $490 \mathrm{~nm}$ using an enzyme-linked immunosorbent assay reader (ELx808; BioTek Instruments, Inc., Winooski, VT, USA).

Colony formation assay. Human prostate cancer cells were cultured in a 6-well plate and transfected with the miR-192 mimics or miR NC. After transfection for $48 \mathrm{~h}$, the cells were re-plated into a 6-well plate in growth medium containing $0.3 \%$ noble agar at 200 cells/well to form natural colonies. After 14 days, the cells were washed with phosphate-buffered saline (PBS), fixed with 4\% paraformaldehyde, and stained with Giemsa (Sigma-Aldrich). The total number of colonies was counted under a microscope (Leica AF6000; Leica, Solms, Germany), and the results were then averaged.

Cell cycle analysis. In the present study, cell cycle distribution (G1, S or G2/M) was detected through flow cytometry. Human prostate cancer cells were transfected with the miR-192 mimics or miR NC for $48 \mathrm{~h}$, harvested, washed, and then fixed with $70 \%$ ethanol. Propidium iodide (PI) $(100 \mu \mathrm{g} / \mathrm{ml}$; Sigma-Aldrich) in PBS containing $10 \mu \mathrm{g} / \mathrm{ml}$ of RNase A was added to the cells and incubated for $30 \mathrm{~min}$ in a dark place at $37^{\circ} \mathrm{C}$. The percentage of cells in each cell cycle phase was 
A

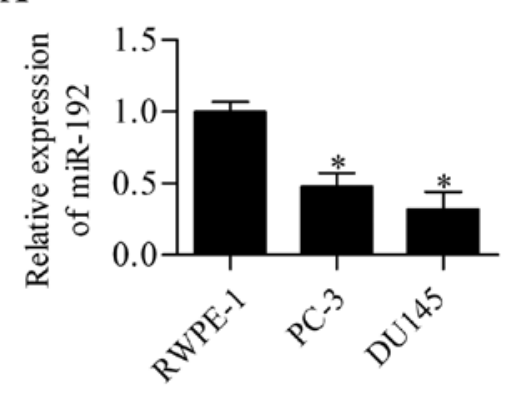

B

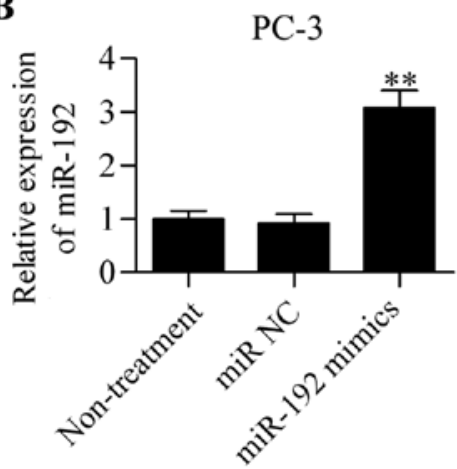

C

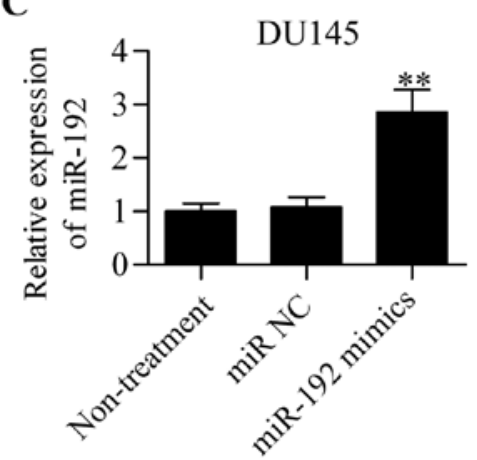

Figure 1. Detection of miR-192 expression in prostate cancer cells. (A) RT-qPCR analysis of miR-192 expression in the prostate cancer cell lines PC-3 and DU145. Human prostate epithelial RWPE-1 cells were used as the control. * $\mathrm{p}<0.05$ vs. RWPE-1. Detection of miR-192 expression levels in (B) PC-3 and (C) DU145 cells transfected with the miR-192 mimics or non-specific miR control (miR NC). After the cells were transfected for 48 h, the cells were harvested and analyzed. Non-treatment, cells which had not received treatment. ${ }^{* *} \mathrm{p}<0.01$ vs. non-treatment or miR NC.

measured using a FACScan flow cytometer (Becton-Dickinson, Franklin Lakes, NJ, USA).

Cell migration assay. In the present study, human prostate cancer cells were transfected with miR-192 mimics or miR NC for $48 \mathrm{~h}$, and the in vitro migration ability was determined using a Transwell chamber (Corning Incorporated, Corning, NY, USA). In brief, $1.0 \times 10^{4}$ cells re-suspended in $200 \mu \mathrm{l}$ serum-free medium were seeded into the upper chamber. Subsequently, $500 \mu \mathrm{l}$ medium containing $10 \%$ FBS was placed in the lower chamber. After the cells were incubated at $37^{\circ} \mathrm{C}$ for $14 \mathrm{~h}$, the cells that remained on the upper chamber were removed using a cotton swab. The cells that had migrated to the lower chamber were subsequently fixed with $10 \%$ methanol for $30 \mathrm{sec}$ and stained with $0.1 \%$ crystal violet (Abcam, Cambridge, UK) for $30 \mathrm{~min}$. The number of cells was then counted under the microscope, and the data obtained in five random fields were averaged.

Dual-luciferase reporter assay. The 3'-UTR of NOB1 containing the predicted binding sites of miR-192 was subcloned into the pGL3 luciferase promoter vector (Promega Corp., Madison, WI, USA). The relevant mutant containing the mutated binding sites of miR-192 was also constructed. The 3'-UTR recombinants of pGL3-NOB1 (100 ng) were cotransfected into the PC-3 cells with miR-192 mimics or miR NC control using Lipofectamine ${ }^{\circledR} 2000$ (Invitrogen) in accordance with the manufacturer's instructions to detect the luciferase reporter activity. pGL3 luciferase reporter vectors contained either the 3'-UTR of wild-type NOB1 or its relevant mutant and miR-192 mimics or non-specific miR control. After the cells were transfected for $48 \mathrm{~h}$, the cells were harvested and lysed. Relative luciferase activity was evaluated using the dual-luciferase reporter system detection method (Promega Corp.) in accordance with standard protocols and the manufacturer's instructions.

Statistical analysis. Data are expressed as the means \pm standard deviation. Statistical significance was calculated through one-way ANOVA, followed by the Bonferroni post hoc test using SPSS version 11.5 (SPSS, Inc., Chicago, IL, USA). A p-value $<0.05$ was considered to indicate a statistically significant difference between groups.

\section{Results}

Expression of miR-192 in prostate cancer cell lines. We evaluated the expression level of miR-192 in PC-3 and DU145 cells through RT-qPCR analysis to determine the potential role of miR-192 in prostate cancer. We noted that miR-192 expression was significantly decreased in the prostate cancer cell lines PC-3 and DU145 compared with that in the prostate epithelial RWPE-1 cells (Fig. 1A). These results indicate that miR-192 is involved in the tumorigenicity of prostate cancer. To explore the potential role of miR-192 in prostate cancer, chemosynthetic miR-192 mimics were used to overexpress miR-192 in the PC-3 and DU145 cells. The results showed that transfection with the miR-192 mimics significantly promoted the expression level of miR-192 in PC-3 (Fig. 1B) and DU145 (Fig. 1C) cells.

Overexpression of miR-192 inhibits the growth of prostate cancer cells. To investigate the effect of miR-192 overexpression on prostate cancer cell proliferation, the PC-3 and DU145 cells transfected with the miR-192 mimics were subjected to MTT assay. miR-192 mimics significantly inhibited the proliferation of PC-3 (Fig. 2A) and DU145 (Fig. 2B) cells compared with the untreated or miR NC-transfected cells. These data indicate that miR-192 plays an important role in regulating the growth of prostate cancer cells.

Overexpression of miR-192 suppresses the colony-forming capacity of prostate cancer cells. To detect whether miR-192 plays an important role in the colony-forming ability of prostate cancer cells, we subsequently performed a colony-forming experiment using PC-3 and DU145 cells which had been transfected with miR-192 mimics. The results showed that miR-192 mimics significantly inhibited the colony formation of the PC-3 (Fig. 3A) and DU145 (Fig. 3B) cells.

Overexpression of miR-192 impairs cell cycle progression. In order to further elucidate the role which miR-192 plays in the tumorigenicity of prostate cancer cells, we detected the effect of miR-192 mimics on cell cycle progression. PC-3 and DU145 cells transfected with miR-192 for $48 \mathrm{~h}$ were subjected to a flow cytometry assay. The results indicated that miR-192 overexpression significantly increased the number 
A

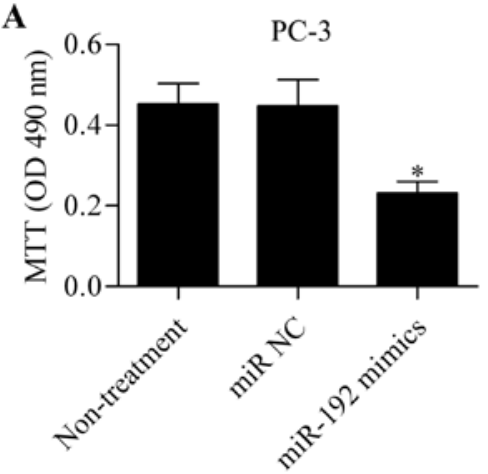

B

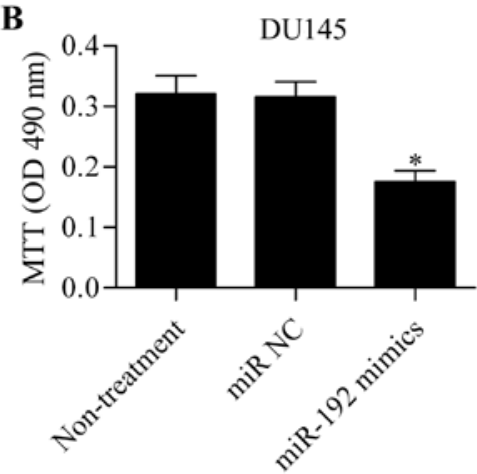

Figure 2. Effect of miR-192 mimics on the growth of prostate cancer cells. miR-192 overexpression inhibited the growth of the (A) PC-3 and (B) DU145 cells, as revealed by MTT assay. The cells were transfected with miR-192 mimics or non-specific miR control (miR NC) for $48 \mathrm{~h}$ and subjected to MTT assay. " $\mathrm{p}<0.05$ vs. non-treatment or miR NC.

A

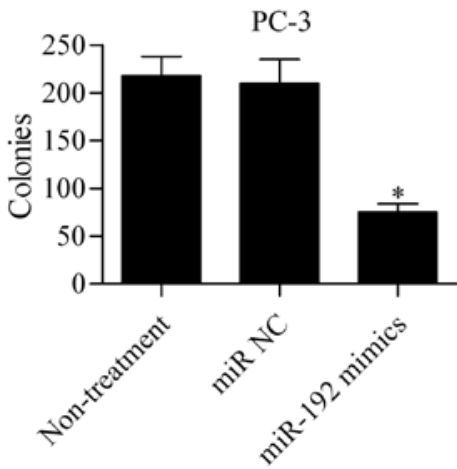

B

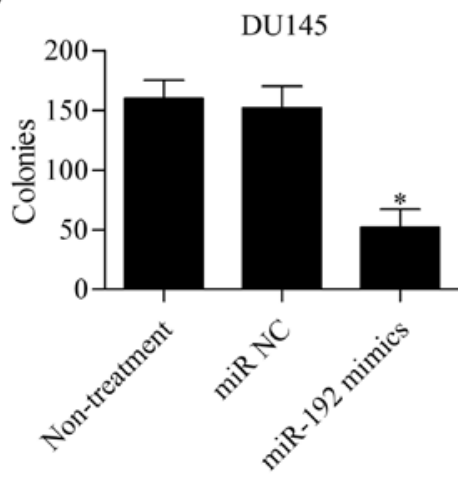

Figure 3. Effect of miR-192 mimics on the colony-forming capacity of prostate cancer cells. The number of colonies in miR-192 mimic- or non-specific miR control (miR NC)-transfected (A) PC-3 and (B) DU145 cells using the Giemsa staining method. " $\mathrm{p}<0.05$ vs. non-treatment or miR NC.

A

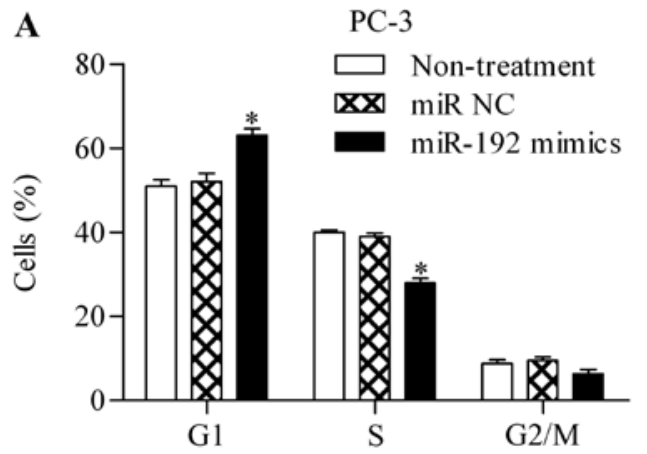

B

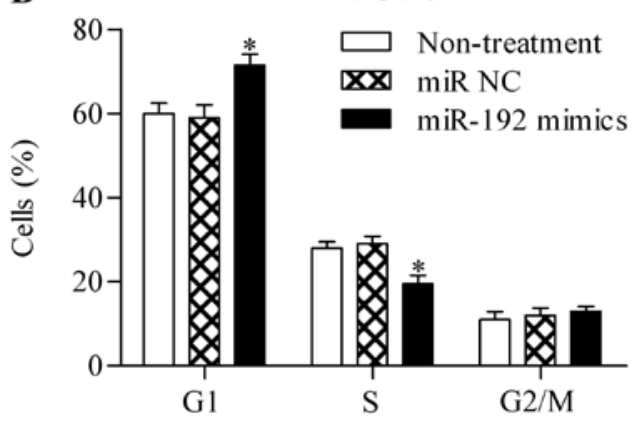

Figure 4. Effect of miR-192 mimics on cell cycle progression. The percentages of (A) PC-3 and (B) DU145 cells in S, G1 and G2/M phases were detected using a flow cytometry assay. After the cells were transfected with miR-192 mimics or non-specific miR control (miR NC) for $48 \mathrm{~h}$, the cells were stained with a PI fluorescent dye and then subjected to the flow cytometry assay. "p<0.05 vs. non-treatment or miR NC.

of cells in the G1 phase whereas miR-192 mimic transfection markedly decreased the proportion of PC-3 (Fig. 4A) and DU145 (Fig. 4B) cells in the S-phase.

Overexpression of miR-192 reduces the migration capacity of prostate cancer cells. To further detect whether miR-192 had an impact on the cell migration of prostate cancer cells, a Transwell migration assay was carried out. We found that miR-192 overexpression considerably reduced the number of PC-3 (Fig. 5A) and DU145 (Fig. 5B) cells that migrated to the lower chamber, implying that miR-192 overexpression reduced the migration capacity of prostate cancer cells.

NOB1 is a direct target gene of miR-192. Taking into consideration that miRs regulate cellular processes through their target genes, we identified the potential target gene of miR-192 through bioinformatics analysis. Notably, we found that NOB1, an oncogene, contained the predicted miR-192 targeting site in the 3'-UTR of NOB1 (Fig. 6A). To verify the direct association between miR-192 and the 3'-UTR of NOB1, pGL3 
A

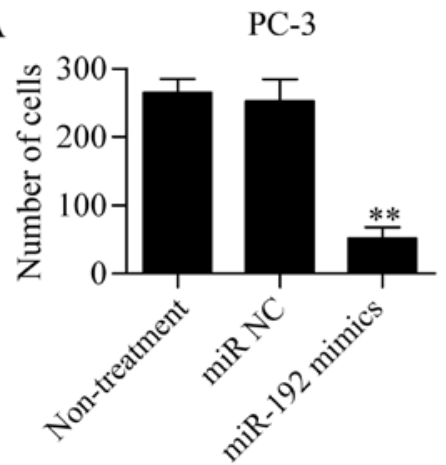

B

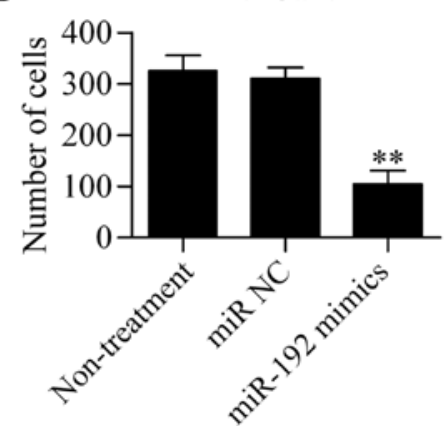

Figure 5. Effect of miR-192 mimics on the migration capacity of prostate cancer cells. (A) PC-3 and (B) DU145 cells were transfected with miR-192 mimics or non-specific miR control (miR NC) for $48 \mathrm{~h}$, and the in vitro migration capacity was determined using the Transwell assay. After $24 \mathrm{~h}$ of incubation, cells that migrated through the membrane were counted with the crystal violet staining method. ${ }^{* *} \mathrm{p}<0.01$ vs. non-treatment or miR NC.

A $\begin{array}{lr}\text { 3'-ccGACAGUUAAGUAUCCAGUc-5' } & \text { Hsa-miR-192 } \\ \text { 5'-aaUUUUUGCUGCUUAGGUCAu-3' } & \text { NOB1 } \\ \text { 5'-aaUUUUUGCUGCUUAGGCACu-3' } & \text { Mutant NOB1 }\end{array}$

B

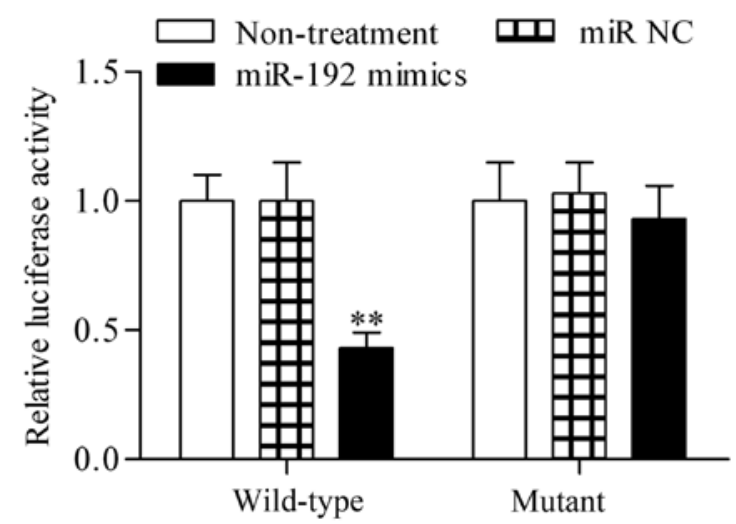

Figure 6. Identification of the miR-192 target sites in the 3'-UTR of nin one binding (NOB1). (A) Schematic of the predicted miR-192 targeting site in the 3'-UTR of NOB1. (B) Dual-luciferase reporter assay was utilized to confirm the direct interaction between miR-192 and the 3'-UTR of NOB1. The PC-3 cells were cotransfected with pGL3 luciferase reporter vectors containing either the 3'-UTR of wild-type NOB1 or its relevant mutant and miR-192 mimics or non-specific miR control (miR NC). After transfection was performed for $48 \mathrm{~h}$, the cells were harvested and lysed. The relative luciferase activity was determined using the dual-luciferase assay detection method ${ }^{* * *} \mathrm{p}<0.01$ vs. non-treatment or miR NC.

luciferase reporter vectors were constructed with the 3'-UTR of the wild-type NOB1 or its relevant mutant. The miR-192 mimics were cotransfected with the reporter vectors into the PC-3 cells. Transfection with miR-192 mimics in the 3'-UTR of wild-type NOB1 significantly reduced relative luciferase activity, whereas transfection with miR-192 mimics had no obvious effect on the corresponding mutant (Fig. 6B). These results showed that NOB1 is a direct target gene of miR-192.

Overexpression of miR-192 reduces the expression of NOB1. In the present study, in order to validate the theory that miR-192 is a regulator of NOB1, we subsequently detected the effect of
miR-192 on mRNA and protein expression levels of NOB1. The results of RT-qPCR analysis demonstrated that miR-192 overexpression caused a marked decrease in the mRNA expression level of NOB1 in PC-3 (Fig. 7A) and DU145 (Fig. 7B) cells. We also noted that the protein expression of NOB1 was significantly reduced by miR-192 overexpression (Fig. 7C and D). These results further confirmed that miR-192 targeted and modulated NOB1 expression.

Overexpression of miR-192 suppresses p38 MAPK expression. To further explore the underlying mechanism of miR-192 in prostate cancer, we detected the effect of miR-192 mimics on p38 MAPK. The results demonstrate that transfection with miR-192 mimic significantly reduced the protein expression level of p38 MAPK in PC-3 cells (Fig. 8A) compared with the miR-NC-transfected group. Similar results were observed in DU145 cells (Fig. 8B).

\section{Discussion}

Previous studies have suggested that abnormally expressed miRs play an important role in the development and progression of cancers $(22,23)$. miR-192 has been reported to act as a tumor suppressor in numerous human cancers $(20,21)$. However, the role of miR-192 in prostate cancers had not previously been documented: to the best of our knowledge, this study is the first to describe the functional significance of miR-192 in prostate cancers. We demonstrated that miR-192 expression was significantly decreased in prostate cancer cell lines. Our results further elucidated that miR-192 overexpression markedly impaired the tumorigenicity of prostate cancer cells. The miR-192 overexpression induced by transfection with miR-192 mimics significantly decreased the proliferation, colony-forming capacity, and migration ability of prostate cancer cells. Moreover, miR-192 overexpression induced cell cycle arrest in the G1 phase. Thus, we suggest that miR-192 serves as a tumor suppressor in prostate cancers.

The NOB1 protein was initially reported to play an essential role in both $20 \mathrm{~S}$ proteasome maturation and protein degradation (6). NOB1 was noted to be extensively involved in various human cancers $(4,7,8)$. NOB1 knockdown has been noted to markedly decrease the colony forming- and proliferative abilities of ovarian cancer cells and induce 
A

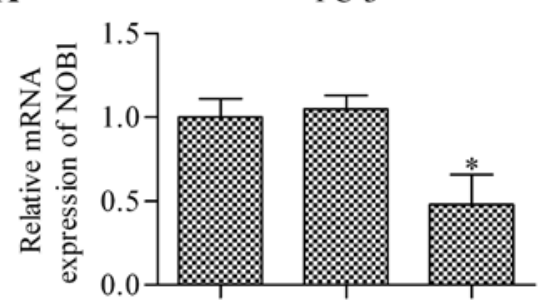

C
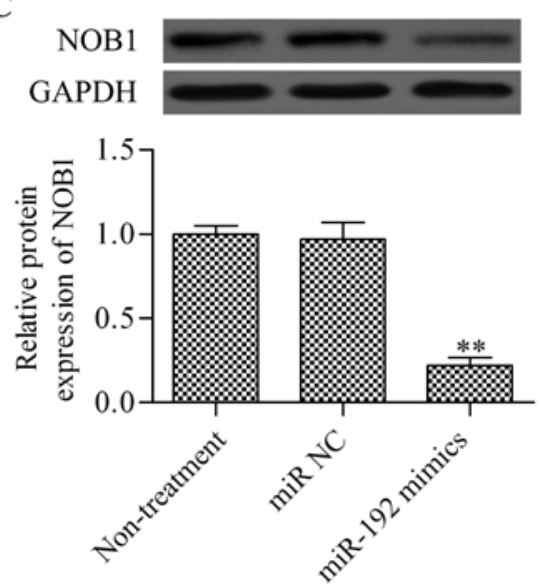

B

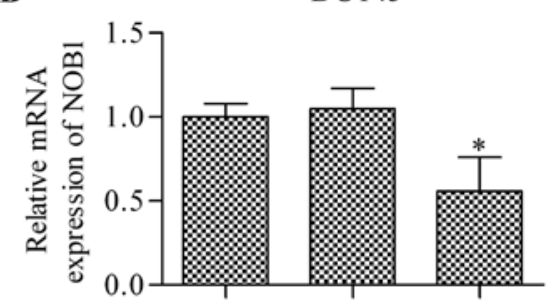

D
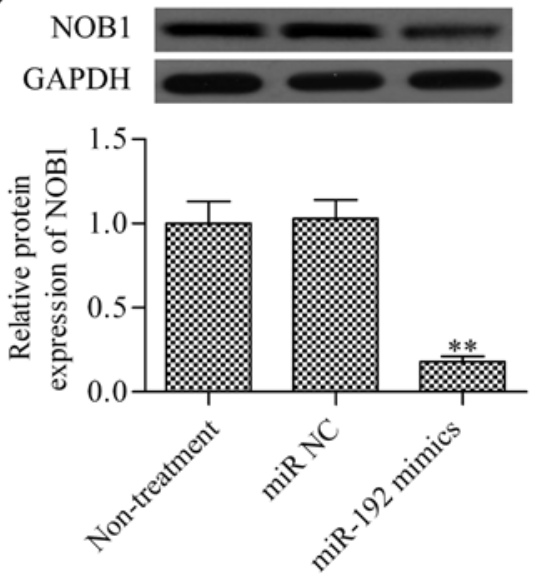

Figure 7. miR-192 regulates nin one binding (NOB1) expression. RT-qPCR was performed to examine the effect of the miR-192 mimic transfection on mRNA expression of NOB1 in (A) PC-3 and (B) DU145 cells. These cells were transfected with miR-192 mimics or non-specific miR control (miR NC) for 48 h. ${ }^{*}$ p $<0.05$ vs. non-treatment or miR NC. Western blot analysis was performed to detect the effect of miR-192 mimic transfection on the protein expression of NOB1 in (C) PC-3 and (D) DU145 cells. Relative protein was quantitatively analyzed using Image-Pro Plus 6.0 and normalized to GAPDH. ${ }^{* *} \mathrm{p}<0.01$ vs. non-treatment or miR NC.

A

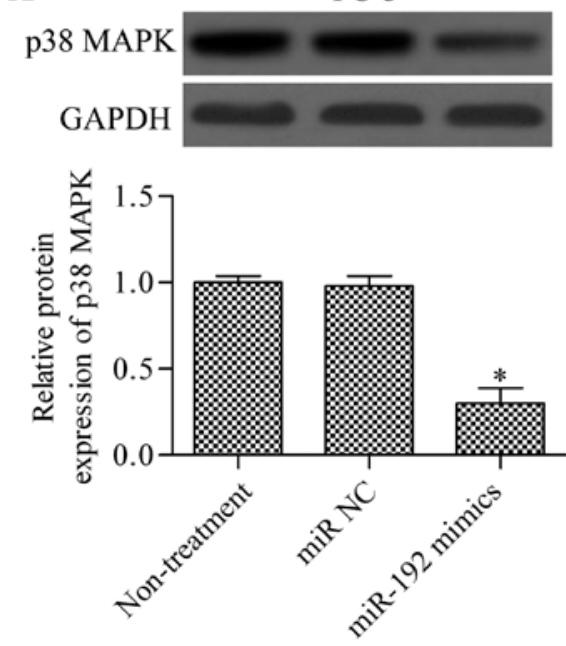

B

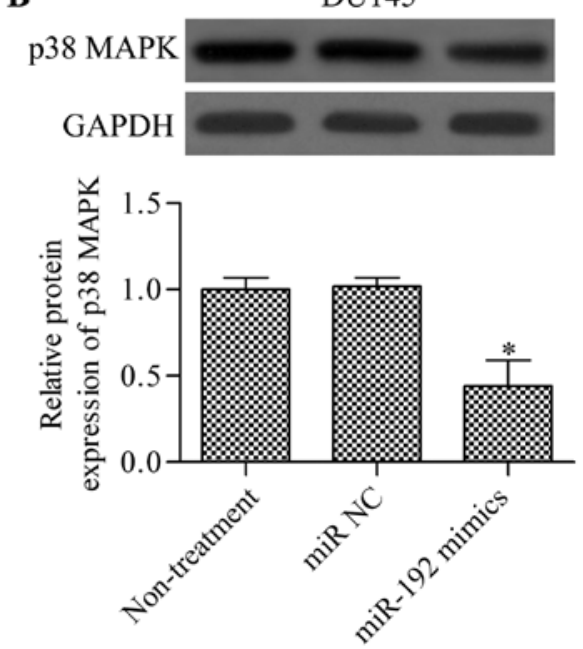

Figure 8. miR-192 regulates p38 mitogen-activated protein kinase (MAPK) expression. Western blot analysis was performed to analyze the effect of miR-192 mimic transfection on the protein expression of p38 MAPK in (A) PC-3 and (B) DU145 cells. Relative protein was quantitatively analyzed using Image-Pro Plus 6.0 and normalized to GAPDH. ${ }^{\text {p }}<0.05$ vs. non-treatment or non-specific miR control (miR NC).

cell cycle arrest in the G0/G1 phase (4). Likewise, the gene silencing ability of NOB1 inhibits the cell growth and tumorformation ability of human hepatocellular carcinoma cells (7). In breast cancer cells, NOB1 knockdown also suppresses the proliferation and growth of cancer cells (8). Studies have also reported that NOB1 was highly expressed in papillary thyroid carcinoma tissues, and silencing NOB1 enhances the antitumor effect of doxorubicin $(9,24)$. NOB1 has also been implicated in the development and progression of non-small-cell lung cancer; it has been suggested that high NOB1 expression acts as a potential indicator of poor prognosis $(10,13,25,26)$. In other types of human cancers, including gliomas (3), osteosarcoma (27), colorectal cancer $(28,29)$, and renal cancer $(30)$, NOB1 has also been found to function as an oncogene. In line with these previous studies, NOB1 has also been studied as an oncogene in prostate cancers. We noted that NOB1 is positively and highly 
expressed in prostate cancer tissues, as has also previously noted (12). More recently, it has been reported that high NOB1 expression in prostate cancer tissues is significantly correlated with distant metastasis and Gleason score (31). These studies also pointed out that NOB1 serves as a potential prognostic indicator of prostate cancer. Furthermore, the gene silencing of NOB1 was found to significantly suppress the tumorigenicity of prostate cancer cells (14). In accordance with these findings, in the present study we found that the NOB1 downregulation induced by miR-192 overexpression also considerably inhibited the tumorigenicity of prostate cancer cells. Our results further confirmed the important role which NOB1 plays in regulating prostate cancer; thus, we suggest that NOB1 serves as a promising molecular target which will assist in the treatment of prostate cancer.

The role which miR-192 plays in several types of human cancer has been widely investigated (20-21,32-34). The tumorigenic or tumor-suppressive function of miR-192 has also been reported in various human cancers. Frequent, high miR-192 expression in gastric cancer is likely involved in the progression of gastric cancer (32). miR-192 overexpression inhibits the proliferation and migration rates in neuroblastoma cells by modulating dicer 1 , ribonuclease type III (Dicer1) expression (33). Geng et al (20) have reported that miR-192 overexpression inhibits the metastasis of colon cancer cells. miR-192 overexpression also decreases cell proliferation and increases cell apoptosis in lung cancer cells by regulating retinoblastoma 1 expression (34). Furthermore, overexpression of miR-192 inhibits the growth of human bladder cancer cells (21). However, whether miR-192 is involved in the development of prostate cancer remains unknown. In the present study, we demonstrated that miR-192 is downregulated in prostate cancer cells, and we thus suggest that it serves as an tumor suppressor in prostate cancer. We then further elucidated that NOB1 is a direct target gene of miR-192 and that miR-192 overexpression inhibited the mRNA and protein expression of NOB1 in prostate cancer cells. Our results indicate that downregulated miR-192 expression in prostate cancer cells accounts for the overexpressed NOB1 which promotes the development and progression of prostate cancer.

miRs are known to be novel regulators of gene expression $(15,16)$. Several studies have evaluated the possibility of decreasing NOB1 expression using specific miRs. For example, it has previously been reported that miR-326 directly targets and regulates NOB1, which inhibited the cell growth of glioma cells associated with the MAPK pathway (35). Moreover, miR-326 has been found to act as a tumor suppressor in colorectal cancer by modulating NOB1 expression (36). In a study on clear cell renal carcinoma, it was noted that miR-646 negatively modulates the expression of NOB1 and suppresses the proliferation and migration of cancer cells; it has been suggested that the MAPK pathway is involved in these processes (37). In the present study, we demonstrated that miR-192 was a novel regulator of NOB1 expression. We also demonstrated that overexpression of miR-192 inhibited the expression of NOB1 and p38 MAPK, which affected the migratory and invasive activity on tumor cells, as was also previously shown (38). The results of our present study are consistent with the reports of Che et al (11), who revealed that silencing of NOB1 inhibited p38 MAPK expression.
In conclusion, our data in the present study demonstrated that miR-192 was downregulated in prostate cancer cells, and also that the restoration of miR-192 inhibited the proliferation, colony-forming capacity, and migratory ability of prostate cancer cells. We also noted that miR-192 overexpression induced cell cycle arrest in the G1 phase. The tumor-suppressive role of miR-192 was likely mediated by regulating NOB1, an oncogene in prostate cancer. Therefore, we suggest that miR-192 serves as a promising therapeutic target to treat prostate cancer.

\section{References}

1. Siegel R, Ma J, Zou Z and Jemal A: Cancer statistics, 2014. CA Cancer J Clin 64: 9-29, 2014.

2. Roy M, Kung HJ and Ghosh PM: Statins and prostate cancer: role of cholesterol inhibition vs. prevention of small GTP-binding proteins. Am J Cancer Res 1: 542-561, 2011.

3. Wang H, Li P and Zhao B: Knockdown of NOB1 expression by RNAi inhibits cellular proliferation and migration in human gliomas. Gene 528: 146-153, 2013.

4. Lin Y, Peng S, Yu H, Teng H and Cui M: RNAi-mediated downregulation of NOB1 suppresses the growth and colony-formation ability of human ovarian cancer cells. Med Oncol 29: 311-317, 2012.

5. Zhang Y, Ni J, Zhou G, Yuan J, Ren W, Shan Y, Tang W, Yu L and Zhao S: Cloning, expression and characterization of the human NOB1 gene. Mol Biol Rep 32: 185-189, 2005.

6. Veith T, Martin R, Wurm JP, Weis BL, Duchardt-Ferner E, Safferthal C, Hennig R, Mirus O, Bohnsack MT, Wöhnert J and Schleiff E: Structural and functional analysis of the archaeal endonuclease Nob1. Nucleic Acids Res 40: 3259-3274, 2012.

7. Lu Z, Guo Q, Shi A, Xie F and Lu Q: Downregulation of NIN/RPN12 binding protein inhibit the growth of human hepatocellular carcinoma cells. Mol Biol Rep 39: 501-507, 2012.

8. Huang WY, Chen DH, Ning L and Wang LW: siRNA mediated silencing of NIN1/RPN12 binding protein 1 homolog inhibits proliferation and growth of breast cancer cells. Asian Pac J Cancer Prev 13: 1823-1827, 2012.

9. Lin S, Meng W, Zhang W, Liu J, Wang P, Xue S and Chen G: Expression of the NOB1 gene and its clinical significance in papillary thyroid carcinoma. J Int Med Res 41: 568-572, 2013.

10. Li Y, Ma C, Qian M, Wen Z, Jing H and Qian D: Downregulation of NOB1 suppresses the proliferation and tumor growth of non-small cell lung cancer in vitro and in vivo. Oncol Rep 31: 1271-1276, 2014.

11. Che JP, Li W, Yan Y, Liu M, Wang GC, Li QY, Yang B, Yao XD and Zheng JH: Expression and clinical significance of the nin one binding protein and $\mathrm{p} 38 \mathrm{MAPK}$ in prostate carcinoma. Int J Clin Exp Pathol 6: 2300-2311, 2013.

12. Liu G, Shen D, Jiao L and Sun Y: Nin one binding protein expression as a prognostic marker in prostate carcinoma. Clin Transl Oncol 16: 843-847, 2014.

13. Liu K, Chen HL, Gu MM and You QS: Relationship between NOB1 expression and prognosis of resected non-small cell lung cancer. Int J Biol Markers 30: e43-e48, 2015.

14. Zhang X, Zhang D, Qu F, Hong Y, Cao J, Pan X, Li L, Huang Y, Huang H, Yin L, et al: Knockdown of NOB1 expression inhibits the malignant transformation of human prostate cancer cells Mol Cell Biochem 396: 1-8, 2014.

15. Bartel DP: MicroRNAs: genomics, biogenesis, mechanism, and function. Cell 116: 281-297, 2004.

16. Winter J, Jung S, Keller S, Gregory RI and Diederichs S: Many roads to maturity: microRNA biogenesis pathways and their regulation. Nat Cell Biol 11: 228-234, 2009.

17. Mendell JT and Olson EN: MicroRNAs in stress signaling and human disease. Cell 148: 1172-1187, 2012.

18. Ranganathan K and Sivasankar V: MicroRNAs - Biology and clinical applications. J Oral Maxillofac Pathol 18: 229-234, 2014.

19. Wang YL, Wu S, Jiang B, Yin FF, Zheng SS and Hou SC: Role of microRNAs in prostate cancer pathogenesis. Clin Genitourin Cancer 13: 261-270, 2015.

20. Geng L, Chaudhuri A, Talmon G, Wisecarver JL, Are C, Brattain M and Wang J: MicroRNA-192 suppresses liver metastasis of colon cancer. Oncogene 33: 5332-5340, 2014. 
21. Jin Y, Lu J, Wen J, Shen Y and Wen X: Regulation of growth of human bladder cancer by miR-192. Tumour Biol 36: 3791-3797, 2015.

22. Goto Y, Kojima S, Nishikawa R, Enokida H, Chiyomaru T, Kinoshita T, Nakagawa M, Naya Y, Ichikawa T and Seki N: The microRNA-23b/27b/24-1 cluster is a disease progression marker and tumor suppressor in prostate cancer. Oncotarget 5: 7748-7759, 2014

23. Augello C, Vaira V, Caruso L, Destro A, Maggioni M, Park YN, Montorsi M, Santambrogio R, Roncalli M and Bosari S: MicroRNA profiling of hepatocarcinogenesis identifies C19MC cluster as a novel prognostic biomarker in hepatocellular carcinoma. Liver Int 32: 772-782, 2012.

24. Liu J, Dong BF, Wang PS, Ren PY, Xue S, Zhang X, Han Z and Chen G: Silencing NOB1 enhances doxorubicin antitumor activity of the papillary thyroid carcinoma in vitro and in vivo. Oncol Rep 33: 1551-1559, 2015.

25. Liu K, Gu MM, Chen HL and You QS: NOB1 in non-small-cell lung cancer: expression profile and clinical significance. Pathol Oncol Res 20: 461-466, 2014.

26. Liu K, Chen HL, Gu MM and You QS: NOB1 expression predicts early response to Cisplatin-based chemotherapy in patients with advanced non-small cell lung cancer. J Chemother: May 13, 2015 (Epub ahead of print).

27. Chen B, Liu J, Wu D, Qin Y, Peng C, Li C and Wang J: Gene silencing of NOB1 by lentivirus suppresses growth and migration of human osteosarcoma cells. Mol Med Rep 9: 2173-2179, 2014.

28. Liu Y, Huang H, Yuan B, Zhuang LY, Luo TP and Zhang Q Lentivirus-mediated knockdown of NOB1 suppresses the proliferation of colon cancer cells. Z Gastroenterol 52: 429-435, 2014.

29. He XW, Feng T, Yin QL, Jian YW and Liu T: NOB1 is essential for the survival of RKO colorectal cancer cells. World J Gastroenterol 21: 868-877, 2015.

30. Jia JW, Liu AQ, Wang Y, Zhao F, Jiao LL and Tan J: Evaluation of NIN/RPN12 binding protein inhibits proliferation and growth in human renal cancer cells. Tumour Biol 36: 1803-1810, 2015.
31. Chen J, Wang J, Cui X, Liu Y, Yin L, Li Y, Chen L, Xu D and Gao Y: Positive nin one binding protein expression predicts poor outcome in prostate cancer. Mol Med Rep 11: 2671-2676, 2015.

32. Xu YJ and Fan Y: MiR-215/192 participates in gastric cancer progression. Clin Transl Oncol 17: 34-40, 2015.

33. Feinberg-Gorenshtein G, Guedj A, Shichrur K, Jeison M, Luria D, Kodman Y, Ash S, Feinmesser M, Edry L, Shomron N, et al: MiR-192 directly binds and regulates Dicerl expression in neuroblastoma. PLoS One 8: e78713, 2013.

34. Feng S, Cong S, Zhang X, Bao X, Wang W, Li H, Wang Z, Wang G, Xu J, Du B, et al: MicroRNA-192 targeting retinoblastoma 1 inhibits cell proliferation and induces cell apoptosis in lung cancer cells. Nucleic Acids Res 39: 6669-6678, 2011.

35. Zhou J, Xu T, Yan Y, Qin R, Wang H, Zhang X, Huang Y, Wang Y, Lu Y, Fu D and Chen J: MicroRNA-326 functions as a tumor suppressor in glioma by targeting the Nin one binding protein (NOB1). PLoS One 8: e68469, 2013.

36. Wu L, Hui H, Wang LJ, Wang H, Liu QF and Han SX: MicroRNA-326 functions as a tumor suppressor in colorectal cancer by targeting the nin one binding protein. Oncol Rep 33 2309-2318, 2015.

37. Li W, Liu M, Feng Y, Xu YF, Huang YF, Che JP, Wang GC, Yao XD and Zheng JH: Downregulated miR-646 in clear cell renal carcinoma correlated with tumour metastasis by targeting the nin one binding protein (NOB1). Br J Cancer 111: 1188-1200, 2014.

38. Li XD, Liu ZY, Chang B, Liu DX, Chen B, Guo C, Wang YG, $\mathrm{Xu}$ JK, Huang DY and Du SX: Panax notoginseng saponins promote osteogenic differentiation of bone marrow stromal cells through the ERK and P38 MAPK signaling pathways. Cell Physiol Biochem 28: 367-376, 2011. 